\title{
Aplicación de tratados sucesivos concernientes a la misma materia. Análisis del artículo 30 de la Convención de Viena sobre Derechos de los Tratados
}

Fecha de recepción: 21 de febrero de 2016

Fecha de aceptación: 27 de mayo de 2016

Doi: 10.12804/revistas.urosario.edu.co/acdi/a.5297

Ana Gemma López Martín*

Resumen: uno de los problemas más complejos que existen en el derecho de los tratados es el relativo a la determinación, acerca de qué tratado es de aplicación, ante la eventualidad de que existan disposiciones convencionales sucesivas que regulen la misma materia y que resulten ser incompatibles. Problema que se solventa cuando los propios tratados tienen prevista esta contingencia en el mismo tratado, pero que se complica ante la ausencia de una cláusula de relación, máxime si no existe identidad de partes entre los distintos tratados sucesivos que regulan la misma materia. El artículo 30 de la Convención de Viena sobre Derecho de los Tratados de 1969 tiene por objeto establecer de forma sistemática las reglas que deben tenerse en cuenta para determinar qué tratado es de aplicación. No obstante, bajo su aparente sencillez, esta disposición esconde una casuística compleja, plagada de remisiones, con innegables deficiencias técnicas congénitas y ausencias destacables — como la regla lex specialis_- El objeto del presente

* Catedrática acreditada de Derecho Internacional Público y RRII de la Facultad de Derecho de la Universidad Complutense de Madrid. Correo electrónico: anagema@der.ucm.es Para citar este artículo: López Martín, A. G. "Aplicación de tratados sucesivos concernientes a la misma materia. Análisis del artículo 30 de la Convención de Viena sobre Derecho de los Tratados", Anuario Colombiano de Derecho Internacional (ACDI), 2017, 10, pp. 51-84. Doi: 10.12804/revistas.urosario.edu.co/acdi/a.5297 
trabajo es analizar con detalle los cinco párrafos en que se desarrolla este artículo, a los efectos de arrojar más luz sobre la materia.

Palabras clave: conflicto de tratados, artículo 103 Carta, cláusulas de relación, lex posterior, derecho de los tratados.

The Application of Successive Treaties Relating the Same Subject-Matter. Analysis of Article 30 of the Vienna Convention on Law of Treaties

Abstract: One of the most complex problems in the Law of Treaties is that relating to the determination of what treaty applies in the event that there are successive treaty provisions regulating the same subject matter which become incompatible. Problem is solved when the treaties themselves have foreseen this eventuality in the same treaty. But that is complicated by the absence of a relationship clause, especially if there is no identity of parties between the successive treaties relating the same subject-matter. Article 30 of the Vienna Convention on the Law of Treaties of 1969 aims to systematically establish the rules to be taken into account in determining what treaty applies. However, under its apparent simplicity, this provision hides a complex casuistry, full of references, with undeniable technical and congenital defects and notable absences such —as the lex specialis rule-. The purpose of this work is to analyze in detail the five paragraphs of the article 30 in order to shed light on this subject.

Key words: Conflict of treaties, article 103 charter, relationship clauses, lex posterior, law of treaties.

Aplicação de tratados sucessivos concernentes à mesma matéria. Análise do artigo 30 da Convenção de Viena sobre direitos dos tratados

Resumo: Um dos problemas mais complexos que existem no direito dos tratados é o relativo à determinação acerca de que tratado é de aplicação ante a eventualidade de que existam disposições convencionais sucessivas que regulem a mesma matéria e que resultem ser incompatíveis. Problema que se resolve quando os próprios tratados têm prevista esta contingência 
no mesmo tratado, mas que se complica ante a ausência de uma cláusula de relação, máxime se não existe identidade de partes entre os distintos tratados sucessivos que regulam a mesma matéria. O artigo 30 da Convenção de Viena sobre o Direito dos Tratados de 1969 tem por objetivo estabelecer de forma sistemática as regras que devem ter-se em conta para determinar que tratado é de aplicação. No entanto, sob a sua aparente simplicidade, esta disposição esconde uma casuística complexa, infestada de remissões, com inegáveis deficiências técnicas congénitas e ausências destacáveis _ como a regra lex specialis_- O objetivo do presente trabalho é analisar com detalhe os cinco parágrafos em que se desenvolve este artigo, aos efeitos de projetar mais luz sobre a matéria.

Palavras-chave: Conflito de tratados, artigo 103 Carta, cláusulas de relação, lex posterior, direito dos tratados.

\section{Introducción}

Entre los problemas jurídicos que se plantean hoy día con mayor vivacidad en la esfera del derecho internacional se sitúa el del conflicto entre normas convencionales sucesivas, que constituye un aspecto particularmente complejo y, en ocasiones, confuso dentro del derecho de los tratados. Este se ha visto acentuado ante el progresivo aumento del volumen convencional acaecido en nuestros días, el cual afecta tanto a sectores en los que ya existe una densa regulación — derechos humanos, derecho del mar o derecho internacional privado, entre otros - como a partes del derecho internacional que son aún fragmentarias y están poco desarrolladas - como ocurre con el sector del medio ambiente-.

$\mathrm{Y}$ es que sucede que, bajo su aspecto formal, cada tratado constituye, con relación a los demás tratados, una entidad aislada, perfecta en ella misma, una especie de mónada supeditada a la regla pacta sunt servanda. Del mismo modo que, desde idéntica óptica, una serie de tratados no constituye - por emplear un lenguaje prestado de las matemáticas - un 'conjunto' ordenado, sino una 'acumulación', lo cual es, sin duda, consecuencia del propio carácter de la sociedad internacional. Es bajo esta perspectiva donde se sitúa la cuestión de la aplicación de los tratados sucesivos concernientes a la misma materia, que presupone la existencia de varios tratados en vigor, algunas de cuyas cláusulas están regulando la misma materia, y respecto de los cuales uno o más Estados sean partes 
en ellos. Ante esta situación, rige la presunción general de validez de tales acuerdos; pese a ello, se llega a contradicciones o incompatibilidades entre dos o más obligaciones sucesivas concurrentes, lo que plantea una cuestión de enorme complejidad. Incluso la propia Comisión de Derecho Internacional (CDI) fue en su día alertada de los riesgos de fragmentación que puede suponer para el derecho internacional que los Estados se hallen vinculados por obligaciones mutuamente excluyentes. ${ }^{1}$

Este aspecto de crisis que afecta al derecho invita al jurista a pronunciarse sobre la norma que deberá ser considerada 'preferente' y a establecer la suerte de las otras normas contradictorias. Esta resulta ser precisamente la tarea que pretendemos abordar a lo largo de estas páginas, tomando como punto de referencia, para ello, el análisis de las soluciones recogidas por la Convención de Viena sobre Derecho de los Tratados del 23 de mayo de 1969 en su artículo 30. Las cuales resultan sencillas en apariencia, pero esconden una compleja estructura plagada de remisiones y reenvíos a otras disposiciones que es preciso desenmarañar, siguiendo para ello el propio iter del artículo 30.

\section{1. Ámbito temporal y material de aplicación del artículo 30}

Tras un complejo proceso de elaboración que arrancó con los trabajos de Lauterpacht, y que continuaron Fitzmaurice y Waldock, los cuales proponían plantear el problema exclusivamente como 'tratados en conflicto', ${ }^{2}$ la CDI optó, al aprobar el proyecto definitivo sobre derecho de los tratados en 1966, por enunciar las reglas que deben regular tan intrincada cuestión de una forma más general y lo hizo bajo el prisma de la 'aplicación de tratados sucesivos concernientes a la misma materia', ${ }^{3}$ tal y como lo recogió finalmente el artículo 30 de la Convención de 1969. Este circunloquio más amplio y ambiguo permite englobar en su ámbito no solo los supuestos

\footnotetext{
1 El Grupo de Estudio sobre Fragmentación analizó dichos riesgos, si bien concluyó que el artículo 30 no planteaba problemas de fragmentación en la medida en que sus disposiciones reflejan consideraciones ampliamente aceptadas y razonables (YbILC, 2004-II, párrs. 332-337).

2 El encabezado del artículo 65 adoptado provisionalmente en 1964 fue "Prioridad en caso de conflictos entre las disposiciones de dos tratados" (Anuario de la CDI, 1964-I, $2^{\mathrm{a}}$ parte, párr. 43).

3 Anuario de la CDI, 1966-II, p. 235 (artículo 26).
} 
de conflictos de tratados, sino cualquier otra relación entre tratados sin necesidad de encontrarse en situación de contradicción.

Sin embargo, tal y como planteó el delegado británico en el primer período de sesiones de la Conferencia de Viena, ${ }^{4}$ la regulación del problema inicial del 'conflicto de tratados' bajo este singular marco hace surgir prima facie las dudas acerca de la exacta conceptualización y alcance de la nueva locución y, más concretamente, de las expresiones 'sucesivos' y 'misma materia'. Circunstancia que hace necesario que nos planteemos un previo análisis conceptual al respecto, a los fines de fijar el concreto ámbito de aplicación del artículo 30.

Dicho análisis debe partir, no obstante, de tener primeramente en cuenta que estamos hablando de 'aplicación', lo que presupone que el problema de determinación de las reglas o criterios por aplicar se produce porque estamos ante tratados en vigor. ${ }^{5}$ Pues, de no ser el caso, no existiría dilema alguno; un conflicto de normas solo puede ocurrir cuando ambas normas estén vigentes y, además, sean aplicables a una situación determinada. Por lo que resulta igualmente imprescindible que al menos un Estado sea parte en los dos tratados; los tratados que vinculan a partes totalmente diferentes no pueden nunca entrar en conflicto.

\subsection{Tratados sucesivos}

El artículo 30 se refiere expresamente a tratados, quedando excluidos a priori de su ámbito de aplicación los supuestos de concurrencia de estos con otras normas internacionales. ${ }^{6} \mathrm{Y}$ con relación a la referencia a 'tratados', debemos retener que el artículo 30 se aplica respecto de todo tipo

\footnotetext{
4 Conferencia de las Naciones Unidas sobre el Derecho de los Tratados, Nueva York, 1969, p. 181.

5 Además, la referencia en el primer párrafo del artículo 30 a "los derechos y obligaciones de los Estados parte" es suficientemente elocuente de que esta cuestión debe plantearse cuando los tratados están en vigor (Paolillo, Felipe, "Article 30", en Corten, O. \& Klein, P. (dirs.), Les Conventions de Vienne sur le droit des traités. Commentaire article par article, Bruylant, Bruxelles, 2006, pp. 1261-1262).

6 Esta circunstancia no excluye, a nuestro juicio, las situaciones de incompatibilidad entre otras 'normas' y, en consecuencia, la posible aplicabilidad por analogía de los criterios fijados por este artículo; máxime la ausencia de jerarquía que existe entre las diferentes normas internacionales.
} 
de tratados, tanto si son multilaterales — ya sean generales, ya sean restringidos- como si son bilaterales. ${ }^{7}$

Ahora bien, debemos cuestionarnos la oportunidad de la utilización del término ‘sucesivos', locución que ha sido objeto de amplia crítica. Pues la idea de la 'sucesividad' constituye un problema por sí misma en el caso de tratados multilaterales en concurrencia, cuando las partes no son las mismas en los diferentes momentos de aplicación de dichos tratados; siendo, además, que la expresión 'tratados sucesivos' puede dar lugar a interpretaciones ambiguas, a los efectos, fundamentalmente, de determinar cuál es el tratado anterior y cuál el posterior. Sin olvidar, como dice Roucounas, que dicha expresión desconoce que pueda haber tratados 'paralelos', esto es, celebrados al mismo tiempo o de forma simultánea, que igualmente entren en conflicto entre sí, y que quedan fuera de la casuística del artículo $30 .^{8}$

Ya durante el primer período de sesiones de la Conferencia de Viena, el delegado británico, Sinclair, ${ }^{9}$ planteó el problema de la expresión 'tratados sucesivos', al exponer que "un tratado firmado posteriormente podría entrar en vigor antes que un tratado firmado con anterioridad, lo que hace cuestionarse cuál es el tratado anterior y cuál el posterior". En este sentido, proponía como ejemplo el siguiente supuesto práctico: una convención multilateral queda abierta a la firma en 1960, el Estado A la ratifica en 1961 y la convención entra en vigor en 1962. En 1963, el Estado A y el Estado B celebran un tratado bilateral sobre la misma materia, que entra en vigor en 1964, después de lo cual, en 1965, el Estado B se adhiere a la convención multilateral. ¿Cuál es el tratado anterior y cuál es el posterior? Desde el punto de vista del Estado A, es anterior la convención multilateral, pero, desde el del Estado B, esa convención es posterior.

En respuesta a esta fundamental cuestión, Waldock sostuvo en su informe ${ }^{10}$ que la fecha importante debería ser la de la adopción y no la de la entrada en vigor, pues el criterio determinante es el de la intención legislativa. La noción subyacente en esta opinión es la de que, cuando el

\footnotetext{
En el mismo sentido, Corten, O. \& Klein, P. (Eds.), The Vienna Conventions on the Law of Treaties. A commentary, vol. I, Oxford University Press, 2011, p. 777.

8 Roucounas, E., "Engagements paralléles et contradictoires", Recueil des Cours, 1987-VI, 206, pp. 35-38.

9 Conferencia de las Naciones Unidas..., op. cit., pp. 181-182.

10 A/CONF.39/C.1/15.
} 
segundo tratado es adoptado, hay una nueva intención legislativa que deberá ser considerada como prevalente sobre la intención manifestada en el instrumento anterior. La opinión de Waldock, no obstante, no ha contado con el unánime respaldo doctrinal; quien más claramente parece compartir este criterio ha sido Sinclair, ${ }^{11} \mathrm{y}$, más recientemente, Aust. ${ }^{12}$

En este sentido, las posiciones sustentadas por los distintos autores sobre este punto se hallan divididas. La razón de esta disparidad la apuntó muy claramente Jenks, para quien, "en el caso de los tratados resulta particularmente difícil determinar cuál es la fecha sustancial al efecto de evaluar la prioridad de la obligación". ${ }^{13}$ Efectivamente, no hay una especial dificultad en el supuesto de conflicto entre instrumentos convencionales claramente concluidos y ratificados en diferentes períodos, pero puede darse el caso de que la cadena de obligaciones basadas en instrumentos en conflicto haya surgido simultáneamente. En tales casos hay distintas fechas por considerar: la fecha de conclusión del tratado; la de apertura a la firma; la de la adopción; la de la manifestación del consentimiento en obligarse por cada una de las partes afectadas; la fecha de entrada en vigor general; la de entrada en vigor particular; o, incluso, la de aplicación provisional, si fuera el caso. La situación puede ser más complicada por las sucesivas revisiones de uno u otro instrumento que puede llegar a ser efectivo para diferentes partes en diferentes fechas.

El problema radica, pues, en la determinación de la 'fecha crítica' que debe tomarse en consideración a los efectos de fijar la posterioridad/ anterioridad de un tratado respecto de otro.

Pero, si analizamos en detalle, tanto el informe de Waldock como la análoga opinión expresada por Sinclair, observamos cómo en su argumentación se plasma una dicotomía: adopción de un tratado, por un lado, y su 'efecto para cada parte individual', por otro. Esta dicotomía responde, tal y como expresa Vierdag, ${ }^{14}$ a la distinción entre dos tipos de conflictos

11 A este respecto, sostiene que 'it seems clear that, in determining which treaty is the 'earlier' and which the 'later', the relevant date is that of the adoption of the text and not that of its entry into force. Adoption of the second treaty manifests the new legislative intent... But, of course, the rules laid down in article 30 bave effect for each individual party to a treaty only as from the date of entry into force of the treaty for that party" (Sinclair, I., The Vienna Convention on the Law of Treaties, Manchester, 1984, p. 98).

12 Aust, A., Modern treaty law and practice, Cambridge University Press, 2007, p. 229.

13 Jenks, L. W., “The conflict of law-making treaties”, BYBIL, 1974-75, p. 444.

14 Vierdag, E. W., "The time of the 'conclusion' of a multilateral treaty: article 30 of the 
de tratados: abstractos y concretos. Los conflictos abstractos tienen lugar en el momento en que ambos instrumentos son adoptados y entran en conflicto el uno con el otro. Mientras que los conflictos concretos solo pueden suceder cuando los Estados afectados han llegado a ser parte en ambos tratados incompatibles. La pregunta que surge entonces es si ambos conflictos caen bajo el ámbito de aplicación de las reglas del artículo 30.

En nuestra opinión, se trata de dos nociones distintas, que abordan problemas diferenciados, y que, por lo tanto, deben mantenerse separadas. Y si nos atenemos tanto al título del artículo 30 como a su contenido, tan solo los conflictos concretos entrarían bajo su consideración, quedando fuera de su ámbito los conflictos abstractos. Por ende, la dicotomía resulta ser inexistente en nuestro concreto ámbito de estudio.

Frente al criterio de la adopción del texto, una mayoría de autores mantiene sobre este punto un parecer diferente, y no plantea - entendemos, acertadamente- la distinción entre conflictos abstractos y concretos. Así, a juicio de Dahl, ${ }^{15}$ la fecha clave es la de la ratificación (o bien cualquier otra forma en que se haya manifestado el consentimiento en obligarse por el tratado en cuestión), pues mantener una fecha anterior podría conllevar, a su juicio, consecuencias irrazonables. Esta opinión, si bien consideramos que resulta más acertada que la expresada por Waldock, no nos parece totalmente asumible, porque la fecha de la manifestación del consentimiento no coincide necesariamente con el despliegue de efectos jurídicos del tratado, que tiene lugar con la entrada en vigor; momento este último a partir del cual el tratado es plenamente operativo, y, por lo tanto, susceptible de ser considerado en su relación con otros tratados anteriores existentes sobre la misma materia.

De esta forma, nos parece más coherente la posición de Soerensen, ${ }^{16}$ quien opta por la fecha de entrada en vigor general del tratado; entiende que la intención de las partes en el momento de la entrada en vigor es la que debe considerarse como decisiva. En esta misma línea se han pronunciado posteriormente otros autores. Es el caso de Czaplinski y Danilenko, ${ }^{17}$

Vienna Convention on the Law of Treaties and related provisions", BYBIL, 1988, pp. 93-95. 15 Dahl, K. N., "The application of successive treaties dealing with the same subject matter", Indian Yearbook of International Affairs, 1974, p. 282.

16 Annuaire de l'IDI, Sesión de Roma, 1973, p. 54.

17 Czaplinski, W. \& Danilenko, G., "Conflicts of norms in international law", NYIL, 1990, p. 19. 
quienes plantean, además, los problemas especiales que pueden causarse por tratados retroactivos, esto es, por aquellos tratados que establecen como fecha de entrada en vigor una fecha anterior a la de la adopción del texto e, incluso, anterior a la fecha de entrada en vigor del tratado que ha sido adoptado primero.

Más contundente y fundamentada es, sin embargo, la opinión de Sciso, ${ }^{18}$ quien añade que el criterio sustentado por Waldock es rechazable porque entra en contradicción con el artículo 18 de la Convención, que obliga a los Estados que han firmado un tratado a reserva de ratificación, o bien han manifestado su consentimiento en obligarse por un tratado que aún no ha entrado en vigor, a no frustrar el objeto y fin de dicho tratado. Se trata de una obligación que es la consecuencia general del principio de buena fe; su contenido y su fundamento descansa en la exigencia de no decepcionar las expectativas de los otros Estados contratantes a que el tratado llegue a ser efectivo.

Esta última argumentación nos parece perfectamente válida, aunque, si nos basamos en el articulado de la propia Convención de Viena, quizá cabría añadir algún otro pilar a esta fundamentación. Así, el artículo 26, que consagra el principio pacta sunt servanda, enuncia la regla de obligatoriedad del tratado limitándolo a los tratados en vigor; mientras el artículo 28 , que disciplina el límite temporal de la eficacia de un tratado en términos de irretroactividad, prescribe como regla general que las disposiciones de un tratado tienen valor vinculante para las partes solo con relación a los hechos o actos posteriores a la entrada en vigor. Además, si nos atenemos al tenor literal del propio artículo 30, este se refiere reiteradamente a las 'partes' y los 'Estados parte', expresiones que deben interpretarse en el sentido establecido por el artículo $2^{\circ}$, párrafo 1 , letra g), de la propia Convención: "Se entiende por 'parte' un Estado que ha consentido en obligarse por el tratado y con respecto al cual el tratado está en vigor".

A los efectos del problema temporal concreto que abordamos, es importante hacer, finalmente, una somera referencia a la calificación jurídica, dentro de este contexto, del fenómeno de la aplicación provisional de los tratados. La posibilidad de aplicación provisional de un tratado o de alguna de sus disposiciones solo puede darse cuando lo disponga el propio tratado o los Estados negociadores así lo hayan convenido de otro modo (Convención de 1969, artículo 25). Esta circunstancia supone la existencia

18 Sciso, E., Gli Accordi Internazionali Confliggenti, Bari, 1986, pp. 68-77. 
de un acuerdo autónomo de naturaleza simplificada, que viene a completar por relación al tratado propiamente dicho; lo que, expresado en otros términos, viene a decirnos que la aplicación provisional entraña la existencia de dos acuerdos que presentan la característica de estar redactados en un instrumento único. A juicio de Picone, ${ }^{19}$ tal acuerdo implica la asunción de obligaciones válidas desde el punto de vista del derecho internacional pero 'no vinculantes'; circunstancia que, en su opinión, se traduce en la inexistencia de sanción contra la violación de dicho acuerdo.

A nuestro juicio, esta opinión es, cuando menos, discutible en la medida en que, a través de la aplicación provisional de un tratado, lo que se está produciendo es la observancia de todas o algunas de sus disposiciones pero antes de su entrada en vigor, hecho que lleva indudablemente aparejado la generación de efectos jurídicos. No obstante, es importante tener presente las especiales características particulares que presenta la institución de la aplicación provisional. Estas se localizan, principalmente, en su naturaleza precaria —es un tratado a término: ya sea por su entrada en vigor definitiva, ya sea por la notificación de un Estado de su intención de no llegar a ser parte- y en el contexto de extremada urgencia en que debe producirse; todo ello sin olvidar la posibilidad de aplicación provisional del tratado multilateral solo entre algunas partes de este, así como el hecho de que el tratado llegue a entrar en vigor respecto a algunas de las partes mientras que otras continúen aplicándolo provisionalmente. La consideración de estas características esenciales en el supuesto de que dicho acuerdo de aplicación provisional entre en conflicto con otro tratado concerniente a la misma materia se traduce en la menor 'resistencia' de las disposiciones de un acuerdo de aplicación provisional frente a las de un tratado formalmente en vigor, circunstancia que debe igualmente retenerse en relación con la importante cuestión de la producción de los efectos jurídicos del supuesto conflicto.

\subsection{Sobre la misma materia}

Por lo que se refiere al ámbito material, la aplicación del artículo 30 se circunscribe a tratados 'concernientes a la misma materia'. Esta parte de la frase fue introducida, como ya hemos apuntado, en el proyecto definitivo

19 Picone, P., L’applicazione in via provisoria degli accordi internażionali, Nápoles, 1973, p. 121. 
de la CDI de 1966. Los trabajos preparatorios no contenían ninguna indicación al respecto.

A lo largo de la Conferencia de Viena, fue el delegado británico, Sr. Sinclair, quien planteó la cuestión del significado de las palabras "misma materia", proponiendo una interpretación restrictiva que excluyera del ámbito de aplicación del artículo 26 (artículo 30) el caso de un tratado de carácter general que abarcara indirectamente el mismo contenido que una disposición particular de un tratado más específico. ${ }^{20}$ Requerida por el Reino Unido la intervención del relator especial de la CDI para aclarar las dudas planteadas, Waldock presentó en la 91ª sesión el correspondiente informe. ${ }^{21}$ En este, y con relación a las palabras "concernientes a la misma materia”, entendía que dicha expresión no comprendía los casos en que un tratado general se opusiera indirectamente a una provisión especial de un tratado, y que en los casos más complejos la interpretación debería orientarse según el principio generalia specialibus non derogat. De esta forma, parece que la existencia de un conflicto tan solo puede producirse respecto de aquellos tratados cuyo objeto presenta "un grado de 'generalidad' comparable".22

No obstante, consideramos que la cuestión no es tan simple como parece a priori, por lo que no puede operarse sin más un criterio de exclusión del artículo 30 — por razón del carácter del propio tratado—, como el propuesto por Waldock, pues a veces los tratados generales contienen reglas más particulares que los tratados específicos.

\footnotetext{
20 Concretamente, celebrándose la $31^{a}$ sesión, puso como ejemplo diferentes instrumentos relativos a los derechos humanos, a saber: los pactos de las Naciones Unidas sobre los derechos humanos de 1966, la Convención Europea sobre Derechos Humanos de 1950, las convenciones especiales de la OIT y de la Unesco relativas a ciertos aspectos de los derechos humanos; y se preguntaba si todos ellos trataban de la misma materia en el sentido del artículo 26 (actual artículo 30). En 1969, el mismo delegado reincidió nuevamente en la cuestión, si bien esta vez propuso como ejemplo una convención sobre la responsabilidad de terceros en materia de energía nuclear - la cual contiene disposiciones sobre la competencia judicial y los efectos de las sentencias dictadas por los tribunales de los terceros Estados-y una convención general relativa al reconocimiento y la ejecución de sentencias extranjeras. En opinión del Sr. Sinclair, no debería considerarse que la primera convención concerniera a la misma materia que la segunda (Conferencia de las Naciones Unidas..., op. cit., pp. 183 y ss.).

21 A/CONF.39/C.1/15.

22 Reuter, P., Introduction an droit des traités, París, 1985, p. 119.
} 
Estamos más de acuerdo con Vierdag ${ }^{23}$ en que se deberá proceder en cada caso concreto a efectuar un test de aplicabilidad para verificar si estamos en presencia de la misma materia. Este autor propone como test-tipo el siguiente: en la medida en que la aplicación de dos reglas a una misma serie de hechos o de acciones conduzca a resultados incompatibles, estaremos seguramente ante una 'misma materia'. Además, a juicio de este autor, no es conveniente aplicar un principio como el de generalia specialibus non derogat, "if the generalia in one instrument are so phrased as to rule out any specialia in another instrument". ${ }^{24}$ En este sentido, este autor propone un ejemplo muy elocuente, tal es la comparativa entre el artículo $19.2 \mathrm{del}$ Pacto Internacional de Derechos Civiles y Políticos de 1966 — que establece la libertad de las comunicaciones-y el artículo 428 del Reglamento de la Conferencia Administrativa Mundial de Radio de 1971 — que somete la concesión de licencias a la necesidad de un previo acuerdo- Si se aplica, sin más, el criterio de la lex generalis según propone Waldock, ello llevaría a considerar al citado Reglamento como lex specialis y, en consecuencia, no cabría aplicar, según su argumento, el artículo 30. Sin embargo, si se observa atentamente el contenido de las respectivas disposiciones puestas como ejemplo, resulta que es más especial la del Pacto que la del Reglamento, por lo que, a nuestro juicio, no puede aplicarse de manera automática el test de exclusión del artículo 30 propuesto por Waldock, pues este no es operativo cuando nos enfrentamos a un supuesto en el que lo que es generalidad en un instrumento particular está igualmente redactado como especialidad en otro general.

Por otra parte, debemos tener en cuenta que el artículo 30 se refiere a la compatibilidad/incompatibilidad entre las disposiciones concretas de los tratados, no se refiere a los tratados en general. Por lo que, situados ante dos tratados respecto de los cuales no hay provisión alguna sobre su relación y que parecen prima facie regir una misma situación, lo que habrá que observar es si el uno afecta al otro. Se considera que le afecta si lo completa, lo excluye, lo amplia, lo restringe, lo hace dudoso. Si los dos tratados pueden ser aplicados cumulativamente, la coexistencia paralela está asegurada. Si no es posible, los modestos medios del artículo 30 entran en juego. En cualquier caso, en ambos supuestos estaríamos en el ámbito de aplicación del artículo 30.

\footnotetext{
23 Vierdag, E. W., “The time of the 'conclusion' of a multilateral treaty...”, op. cit., p. 100. 24 Ibid., p. 100.
} 


\section{E1 artículo 103 de la Carta de las Naciones Unidas como primer criterio de aplicación}

Tal y como reza el párrafo primero del artículo 30, las reglas sobre prioridad en la aplicación de tratados sobre la misma materia se entienden bajo reserva del artículo 103 de la Carta de las Naciones Unidas. Esta disposición establece la primacía de las obligaciones impuestas por la Carta sobre toda obligación contraída en virtud de cualquier otro tratado internacional.

La inclusión de esta referencia especial al artículo 103 de la Carta se justifica por parte de la CDI atendiendo a "la importancia en el derecho internacional moderno de la Carta de las Naciones Unidas" y al hecho de que "los Estados miembros de las mismas constituyen una parte importante de la comunidad internacional" ${ }^{25}$ — dato este último que se refuerza hoy día por su composición cuasiuniversal-.

Sea como fuere, es evidente que se trata de una reserva a la disciplina general del artículo 30, sobre la cual es necesario detenerse someramente.

En primer lugar, debemos tener presente que el artículo 103 plantea una relación de jerarquía entre dos categorías de normas: i) "las obligaciones de los Estados miembros contraídas en virtud de la Carta", que priman sobre ii) "sus obligaciones en virtud de todo otro acuerdo internacional".

i) Por lo que se refiere a la primera categoría, conviene destacar el dato de que el artículo 103 no dice que solo la Carta deberá prevalecer, sino que se refiere a las obligaciones contraídas en virtud de ella ("obligations under the Charter" en inglés, y "en vertu de la présente Charte” en francés). La cuestión que se plantea entonces es la de delimitar los tipos específicos de obligaciones que se imponen a los Estados miembros en virtud de la Carta, y que quedarían sometidos al artículo 103. Está claro que esta fórmula incluye todas las obligaciones que resultan directa e inmediatamente de la Carta, tales como la obligación de solucionar las controversias de forma pacífica o la prohibición de la amenaza o el uso de la fuerza. Sin embargo, el artículo 103 va más allá, y, en este sentido, habría que referirse a las decisiones

25 Anuario de la CDI, 1966-II, p. 235. Hemos de advertir, no obstante, que esta mención expresa al artículo 103 de la Carta de la ONU supone una redundancia respecto de lo ya dispuesto en el artículo $5^{\circ}$ de la propia Convención que deja a salvo de esta toda norma pertinente de una organización internacional. 
obligatorias de los órganos de la ONU, es decir, el derecho derivado de las Naciones Unidas. Aquí la situación queda menos clara, debiendo distinguirse tres tipos de decisiones:

1. Decisiones obligatorias externamente. Tales son las decisiones del Consejo de Seguridad relativas al mantenimiento de la paz y seguridad internacionales (capítulo VII de la Carta), ${ }^{26}$ así como las sentencias de la Corte Internacional de Justicia (CIJ) en el ejercicio de su función contenciosa, puesto que crean igualmente obligaciones para las partes (artículo 94.1 de la Carta). Ambas caen, claramente, dentro del artículo 103.

2. Decisiones internas obligatorias, pero que tienen efectos externos; este segundo tipo podríamos situarlo también en el ámbito de operación del artículo 103. La CIJ ha reconocido que la Asamblea General puede adoptar decisiones internas con efectos externos, ${ }^{27}$ como son, por ejemplo, la suspensión de derechos y privilegios de los miembros, expulsión de estos y cuestiones presupuestarias.

3. Decisiones externas que son en principio no obligatorias, pero que bajo ciertas circunstancias pueden llegar a tener efecto obligatorio. En términos generales, esta regla se aplica a las recomendaciones de la Asamblea o del Consejo de Seguridad (capítulo VI). Sin embargo, ciertas circunstancias pueden cambiar el efecto de dichas recomendaciones: pueden ser aceptadas expresamente por los Estados miembros, dándole así efecto jurídico; puede tratarse de recomendaciones que

26 El propio Consejo de Seguridad ha invocado el artículo 103 para reafirmar la primacía de sus resoluciones — dictadas en el marco del capítulo VII de la Carta — sobre cualquier otro compromiso convencional. Así, ha ocurrido, por ejemplo, en la Resolución 670 (1990), o en las resoluciones 748, 733 y 788 (1992). En idéntico sentido se ha pronunciado la CIJ en el asunto relativo a las cuestiones de interpretación y de aplicación de la Convención de Montreal de 1971 resultante del incidente aéreo de Lockerbie (Libia/Reino Unido y Libia/EE. UU.), al proclamar la primacía de las resoluciones del Consejo de Seguridad dictadas en el contexto del capítulo VII —resoluciones 748 (1992) y 883 (1993), sobre la referida Convención, con base en el artículo 103 (ICJ Reports, 1992, pp. 16 y 126)—.

27 Así lo reconoció en el dictamen consultivo relativo a ciertos gastos de las Naciones Unidas (ICJ Reports, 1962, pp. 151-163), así como en el asunto sobre Namibia (ICJ Reports, 1971, pp. 16-50). 
demuestren y definan la existencia de derecho consuetudinario (codificación), o, incluso, que creen derecho internacional público general —como la Resolución 1514 (XV)—. No obstante estos casos excepcionales, este tipo de decisiones no entran en la estructura del artículo 103, que se refiere a obligaciones contraídas 'en virtud de la Carta', y no por otras 'circunstancias'.

ii) En segundo lugar, el artículo 103 se refiere a la primacía respecto de obligaciones convencionales contraídas por los Estados miembros, por lo tanto, no se está apuntando al tratado mismo, sino solo a las obligaciones que derivan de este o a las que engendra dicho tratado. Ahora bien, una cuestión que no aclara el artículo 103 es a qué tratados se refiere: ¿a los anteriores a la Carta? ¿A los posteriores? Tal intencionada laguna implica su aplicabilidad, tanto a los tratados concluidos con anterioridad a la Carta como a los que tengan lugar con posterioridad.

Otra cuestión importante sobre la que tampoco dice nada, expresamente, el artículo 103 es la relativa a las consecuencias de la prioridad de la Carta. Concretamente, si los acuerdos incompatibles deben ser considerados nulos o meramente suspendidos. Tampoco regula cómo deben abordarse los tratados concluidos con Estados no miembros de la organización. La respuesta a esta cuestión debe efectuarse analizando separadamente cada una de las posibles situaciones conflictivas.

a) Tratados anteriores entre Estados miembros. Este supuesto es muy claro, pues, además del artículo 103, rige la aplicación del principio general lex posterior derogat prior. Por lo que la solución sería idéntica a la aplicación de la regla subsidiaria del párrafo 3 del artículo 30: prevalece el tratado posterior y el anterior solo se aplica en la medida en que sea compatible con el posterior. ${ }^{28}$

b) Tratados posteriores entre Estados miembros. Aquí es donde realmente despliega todos sus efectos la cláusula de primacía del artículo 103 - que constituye una excepción al principio de

28 El Consejo de Seguridad declaró en 1947 que el tratado anglo-egipcio de 1936 y el acuerdo franco-británico de 1945 eran contrarios a la Carta y, por ello, caducos (Séances $d u$ Conseil de Sécurité, 5 de agosto-10 de septiembre de 1947). 
lex posterior-, siendo de aplicación respecto de todo tipo de tratados celebrados entre Estados miembros de la ONU, tanto bilaterales como multilaterales.

A este respecto, la pregunta que se nos suscita es ¿qué ocurre con esos tratados que son incompatibles con la Carta según los términos del artículo 103? En primer lugar, debemos indicar que no existe verdaderamente un 'procedimiento' para declarar la incompatibilidad; nada dice la Carta al respecto, tampoco se prevé ningún procedimiento, ni se menciona cuál será el órgano de las Naciones Unidas competente para determinar la incompatibilidad entre las obligaciones en caso de producirse una situación de conflicto. Es una cuestión por resolver por la propia práctica de la organización, de la cual cabe extraer la conclusión de que el artículo 103 puede ser — y ha sido- invocado, ya sea de forma principal o de forma incidental, en el marco de los debates tanto del Consejo de Seguridad como de la Asamblea General2 ${ }^{29}$ (o de sus órganos subsidiarios), así como en el contexto de un procedimiento ante la CIJ. ${ }^{30}$ Pero pensamos que quizá podría llegar a ser igualmente factible que fuera el Secretario General, órgano encargado de registrar los tratados según el artículo 102 de la Carta, el que de forma automática apreciara dicha incompatibilidad al proceder a dicho registro, detectándolo, así, antes de su plena efectividad.

Por lo que se refiere al problema de los efectos jurídicos, no parece admisible la tesis de la nulidad, que entendemos es tan solo aplicable en el supuesto de que la obligación contraída por virtud de la Carta sea una norma de ius cogens; pero, entonces,

29 Así, en el contexto de la Asamblea General, el caso más interesante fue el de la denuncia presentada por la URSS a través de un memorándum de 30 de marzo de 1949, en el que acusaba a los signatarios del Tratado del Atlántico Norte de haber violado la Carta de las Naciones Unidas; estos respondieron ante la Asamblea General en una nota del 2 de abril de 1949, y tras largos debates se declaró la inexistencia de incompatibilidad entre ambos textos (Beckett, W. E., The North Atlantic Treaty, the Brussels Treaty and the Charter of the United Nations, London, 1950, pp. 1-35).

30 A este respecto, la CIJ afirmó, en el asunto relativo a las actividades militares y paramilitares en y contra Nicaragua (excepciones preliminares), con relación al acuerdo regional del proceso de Contadora, que "... all regional, bilateral, and even multilateral, arrangements that the parties to this case may have made, touching on the issues of settlement of disputes or the jurisdiction of ICJ, must be made always subject to the provisions of article 103 of the Charter" (ICJ Reports, 1984, p. 440). 
aplicaríamos el artículo 53 o el artículo 64 de la Convención de Viena sobre Derecho de los Tratados, según sean los tratados posteriores o anteriores, respectivamente, y no el artículo 103 de la Carta. Este último no se pronuncia en términos de invalidez, sino de prevalencia en la aplicación. Se trata, como ya hemos apuntado, de una cláusula conflictual contenida en un tratado, que tiene por finalidad asegurar la observancia de la Carta cuando esta entra en conflicto con otro tratado; este tipo de cláusulas de primacía (como veremos en el próximo apartado) no tienen como efecto la 'anulación' del otro tratado, sino la aplicación preferente del que las contiene. Por lo que, a nuestro juicio, el artículo 103 debe interpretarse de forma que, en caso de conflicto con la Carta, el tratado que contenga la estipulación incompatible deviene 'inaplicable', pero solo respecto de la disposición o disposiciones incompatibles, pues, tal y como hemos expuesto, el artículo 103 plantea el conflicto entre 'obligaciones', por lo que la solución de 'inefectividad' no es extensible a la totalidad del acuerdo, sino solo a las obligaciones derivadas de él e incompatibles.

Debemos indicar, igualmente, que el artículo 103 ha sido invocado por algunos Estados tanto en el marco del Tribunal Europeo de Derechos Humanos como ante el Comité de Derechos Humanos a los efectos de justificar la suspensión temporal de ciertos derechos humanos, en la medida en que esta se ampara en resoluciones del Consejo de Seguridad adoptadas en el marco del capítulo VII de la Carta que priman sobre los correspondientes tratados de derechos humanos que los recogen - Convenio de Roma sobre Derechos Humanos y Libertades Fundamentales de 1950 y Pacto Internacional de Derechos Civiles y Políticos de 1966, respectivamente— ${ }^{31}$ Argumentación lógica, pues el artículo 103 no excepciona ninguna categoría de tratados; la única categoría de derechos que sí supondría una excepción a la aplicabilidad del artículo 103 en este contexto serían los que tuvieran naturaleza de ius cogens.

31 Tal ha sido el caso del Reino Unido — caso Al-Jedda — y de Suiza — caso Nada — ante el TEDH; y de Bélgica — casos Sayadi y Vinck — ante el Comité (ver la referencia en Paulus, L., "Article 103", en Simma, Bruno (ed.), The Charter of the United Nations. A commentary, vol. II, $3^{\text {a }}$ ed., Oxford University Press, 2012, pp. 2120-2121 y 2129). 
c) Tratados concluidos por Estados miembros con Estados no miembros de la ONU. El artículo 103 se refiere a obligaciones convencionales contraídas por los Estados miembros, sin hacer mención expresa al problema concreto de que tales obligaciones hayan sido contraídas respecto de Estados no miembros. ¿Es aplicable a estos la primacía de la Carta que consagra el artículo 103? Sin duda, se trata de la cuestión más controvertida. La solución al problema de obligaciones conflictivas resulta clara cuando nos enfrentamos a normas de ius cogens contenidas en la Carta, pues estas son claramente oponibles a Estados no miembros y conllevarían la aplicación de los artículos 53 y 64 de la Convención de Viena. Pero, aparte de los casos que suponen colisión con el ius cogens, la situación jurídica de los tratados con terceros Estados no está clara, y la doctrina está dividida; si bien la opinión mayoritaria parece ser la de la aplicación del artículo 103 a los Estados no miembros, basándose para ello en la consideración de la Carta como 'constitución' de la comunidad internacional, incluso con carácter imperativo. ${ }^{32}$

A este respecto, creemos importante retener en este punto la operatividad del principio pacta tertiis nec nocent nec prosunt, que impide la extensión a terceros de los derechos y obligaciones derivadas de un tratado sin su consentimiento. Por lo que, en nuestra opinión, salvo el caso de obligaciones erga omnes y los supuestos previstos por el artículo 2.6 y el capítulo VIII de la Carta, en caso de conflicto entre una obligación contraída en virtud de la Carta y otra obligación derivada de un acuerdo internacional entre un Estado miembro y otro Estado no miembro, si bien el Estado miembro

32 Es el caso, entre otros, de Bernhardt, R., "Article 103", en Simma, Bruno (ed.), The Charter of the United Nations: a commentary, Oxford University Press, 1994, pp. 1122-1123; Conforti, B., "Organizzazione delle Nazioni Unite", Enciclopedia del diritto, vol. XXXI, Torino, 1981, pp. 267 y ss.; Giardina, A., “The Egytptian-Israeli Peace Agreement”, Italian Yearbook of International Law, 1979, pp. 21 y ss.; o Meron, T., "On a Hierarchy of international human rights", AJIL, 1986, pp. 1 y ss. (sobre esta particular cuestión, ver en más detalle el trabajo de Kolb, Robert, "L'article 103 de la Charte des Nations Unies", Recueil des Cours, 2013, 367, pp. 235-240). Desde nuestro punto de vista, no creemos que exista una base suficiente para poder afirmar la naturaleza imperativa de toda la Carta, en la medida en que tal naturaleza precisa de su aceptación como tal por toda la comunidad internacional en su conjunto — según dispone el artículo 53 de la Convención de Viena de 1969—, lo cual dista mucho de haberse conseguido. 
estará obligado por el artículo 103 de la Carta a aplicar las obligaciones que devienen de esta — generándose los efectos que acabamos de indicar-, si así lo hace, el Estado tercero podrá reclamar por los daños que deriven de lo que claramente es un incumplimiento convencional. Obviamente, esta solución no sería factible en el supuesto de que la obligación contraída por la Carta se hubiere erigido en una norma consuetudinaria y fuera oponible al Estado no miembro por esa vía.

\section{La existencia de cláusulas convencionales de relación entre tratados}

Las reglas de prioridad enunciadas en el artículo 30 tienen carácter supletorio, en la medida en que solo se aplican cuando los Estados no hayan incluido expresamente en los tratados cláusulas que regulen la relación entre distintos instrumentos convencionales. Es decir, lo primero que ha de hacerse es atender a la voluntad de los Estados negociadores a este respecto recogida en los propios tratados. En este sentido, hemos de advertir que es un hecho constatado que de forma cada vez más frecuente la concurrencia de disposiciones convencionales aparece resuelta en el texto de uno o de varios de los tratados sucesivos, lo cual tiene lugar a través de las llamadas cláusulas de relación.

Sobre este particular, podemos encontrarnos con diferentes tipos de cláusulas:
a) de exclusión de las disposiciones de otros tratados;
b) de prioridad de sus disposiciones sobre las divergentes de otros tratados;
c) de recepción, a efectos interpretativos, o de complemento de otros tratados;
d) de subordinación a otro tratado;
e) de compatibilidad con otros tratados.

\subsection{Las cláusulas de relación recogidas en el artículo 30.2}

Según el tenor del párrafo 2 del artículo $30,{ }^{33}$ de las anteriormente referidas cláusulas de relación, este solo recoge las dos últimas, esto es, las

33 Dice expresamente el artículo 30.2 de la Convención: "Cuando un tratado especifique 
de subordinación y las de compatibilidad con otros tratados. Estaríamos hablando de cláusulas que conceden prioridad vis-à-vis a otro tratado distinto del que las contiene.

De esta forma, en el marco del artículo 30.2, encontramos una primera serie de cláusulas, las de subordinación, que suelen emplear términos tales como 'prevalece', 'está subordinada', 'se aplicará', 'no afectará'. Estas tienden a hacer primar a otro tratado, ya sea anterior, ya sea posterior. Los dos tratados coexisten, pero aquel al que reenvía la cláusula prima sobre el otro en caso de conflicto.

Un ejemplo de este tipo es la cláusula contenida en el artículo 73 de la Convención de Viena sobre Relaciones Consulares de 1963: "1. Las disposiciones de la presente Convención no afectarán a otros acuerdos internacionales en vigor entre los Estados que sean parte en los mismos".

También es el caso del artículo $3^{\circ}$ de la Convención de las Naciones Unidas sobre el Derecho de los Usos de los Cursos de Agua Internacionales para Fines Distintos de la Navegación, de 1997: "1. Salvo acuerdo en contrario, nada de lo dispuesto en la presente Convención afectará a los derechos u obligaciones de un Estado del curso de agua derivados de acuerdos que hayan estado en vigor respecto de él en la fecha en que se haya hecho parte en la presente Convención".

Es posible, igualmente, que la cláusula se refiera a la primacía de los acuerdos futuros. Es el caso del artículo 17 del Acuerdo entre la República de Gambia y la República de Senegal sobre el Establecimiento de una Confederación Senegambesa: "Each Confederated State may conclude International Agreements in accordance with its constitutional requirements... if any disagreement arises between this Agreement and any other international obligation, the provisions of this Agreement shall prevail". ${ }^{34}$

La segunda fórmula del artículo 30.2 se refiere a las cláusulas de compatibilidad, que contienen fórmulas como 'es compatible', 'no es contrario', 'es sin perjuicio' o 'no deroga' (por recoger algunas de las expresiones más corrientes), con relación a otro tratado, que es normalmente designado de forma expresa, o con relación a aquellos tratados concernientes a la misma materia. Está claro que estas cláusulas declaran la coexistencia de ambos tratados, y la inexistencia de conflicto entre sus disposiciones. Aquí los ejemplos son muy numerosos.

que está subordinado a un tratado anterior o posterior o que no debe ser considerado incompatible con ese otro tratado, prevalecerán las disposiciones de este último".

34 UN Treaty Series, vol. 1261, p. 332. 
Artículo 25 de la Convención de las Naciones Unidas contra el Tráfico Ilícito de Estupefacientes y Sustancias Psicotrópicas, 19 de diciembre de 1988: "Las disposiciones de la presente Convención no derogan ningún derecho u obligación que la Convención de 1961 tal y como ha sido modificada, o la Convención de 1971 reconocen o imponen a las partes de la presente Convención".

Artículo $4^{\circ}$ del Acuerdo sobre la Aplicación de las Disposiciones de la Convención de las Naciones Unidas sobre el Derecho del Mar de 1982 Relativas a la Conservación y Ordenación de las Poblaciones de Peces Transzonales y las Poblaciones de Peces Altamente Migratorios, 4 de diciembre de 1995: "Ninguna disposición en el presente Acuerdo se entenderá en perjuicio de los derechos, la jurisdicción y las obligaciones de los Estados con arreglo a la Convención. El presente Acuerdo se interpretará y aplicará en el contexto de la Convención y de manera acorde con ella".

Artículo XIV de la Convención de Washington sobre el Comercio Internacional de Especies de Fauna y Flora Salvaje en Peligro de Extinción, 3 de marzo de 1973: "4. Las disposiciones de la presente Convención no afectan a las disposiciones o las obligaciones derivadas de todo otro tratado, convención o acuerdo internacional concluido o por concluir entre Estados relativo a la creación de una unión o de una zona comercial regional que comporte el establecimiento o el mantenimiento de controles comunes aduaneros y la supresión de controles interiores".

Debemos indicar que, aun cuando expresamente no se recoge en el literal del artículo 30.2, también quedaría enmarcada en este contexto una tercera fórmula de cláusula de relación: aquella que refiera que el tratado 'completa' las disposiciones de otro tratado. Y creemos que esto es posible, pues la filosofía que subyace en el párrafo 2 es la de permitir aquellas cláusulas que no lesionan los principios generales del derecho de los tratados y que, por lo tanto, evitan el conflicto entre tratados concurrentes. Las cláusulas de complemento se ajustan a dicha lógica. Veamos algunos ejemplos.

Artículo 73.2 de la Convención de Viena sobre Relaciones Consulares de 1963: "Ninguna de las disposiciones de la presente Convención impedirá que los Estados concierten acuerdos internacionales que confirmen, completen, extiendan o amplíen las disposiciones de aquellas".

Artículo VIII del Acuerdo entre los Estados Unidos y la URSs de 1990 sobre la destrucción y no producción de armas químicas y sobre medidas para facilitar la Convención Multilateral sobre Armas Químicas de Banning, 1 de junio de 1990: "Otherwise, the provisions of this Agreement 
shall supplement the provisions of the multilateral convention in its operation between the parties". 35

\subsection{Otras cláusulas de conflicto}

Ya sea porque pueden quedar subsumidas en el régimen general de la lex posterior recogido en el párrafo 3 del artículo 30, ya sea porque se sitúen en el contexto de la terminación de tratados, o bien por su nula eficacia frente a la autonomía de la voluntad de las partes, lo cierto es que a la hora de referirse a la intención de las partes manifestada en el propio tratado, como primer parámetro en la solución de concurrencia y conflicto de tratados sobre una misma materia, la CDI decidió intencionadamente suprimir cualquier referencia expresa a otros posibles tipos de cláusulas de relación. Es el caso de las cláusulas de primacía y de las de exclusión de las disposiciones de otro tratado, que otorgan prioridad al tratado que las contiene sobre otro, ya sea anterior o posterior — de las que el artículo 103 de la Carta, ya visto, es un buen ejemplo-.

Tal es el caso del artículo 311 de la Convención de las Naciones Unidas sobre el Derecho del Mar (CNUDM) del 10 de diciembre de 1982: “1. Esta Convención prevalecerá en las relaciones entre los Estados parte, sobre las Convenciones de Ginebra sobre el Derecho del Mar, de 29 de abril de 1958".

Refiriéndose a esa misma Convención, el posterior acuerdo relativo a la parte XI, de 1994, regula en su artículo $2^{\circ}$ la relación entre ambos tratados, primando al acuerdo en caso de conflicto: “1. [...] En caso de haber discrepancia entre este Acuerdo y la parte XI, prevalecerán las disposiciones de este Acuerdo".

Artículo 82 de la Convención de Chicago de 1944 sobre Aviación Civil Internacional: "Los Estados contratantes acuerdan que el presente convenio abroga todas las obligaciones y entendimientos mutuos que sean incompatibles con sus disposiciones y se comprometen a no contraer tales obligaciones o entendimientos".

35 Citado por Roucounas, E., “Engagements paralléles et contradictoires...”, op. cit., p. 90. 


\section{Las reglas residuales del artículo 30}

Aunque no aparezca expresado como tal, las reglas del artículo 30 - que permiten determinar cuál de los tratados sucesivos sobre la misma materia debe aplicarse - tienen carácter residual, ${ }^{36}$ pues solo operan en ausencia de cláusula de conflicto. Tales reglas distinguen, en párrafos diferenciados, dos posibles situaciones: identidad (párrafo 3) o no identidad de partes (párrafo 4) en dichos tratados; en la medida en que las soluciones que se articulan para cada uno de los supuestos son claramente distintas. Y ello es así en consonancia con los dos principios fundamentales del derecho de los tratados que rigen la materia: pacta sunt servanda y pacta tertiis nec nocent nec prosunt.

\subsection{Identidad de partes en los tratados sucesivos}

Tal situación es la regulada por el párrafo 3 del artículo 30: "Cuando todas las partes en el tratado anterior sean también partes en el tratado posterior pero el tratado anterior no quede terminado ni su aplicación suspendida conforme al artículo 59, el tratado anterior se aplicará únicamente en la medida en que sus disposiciones sean compatibles con las del tratado posterior".

Del tenor literal de este párrafo se desprende claramente la consagración, por parte de la Convención, de la máxima lexposterior derogat priori como criterio de solución para el caso en que todas las partes en un tratado concluyan, posteriormente, un nuevo tratado sobre la misma materia sin poner fin al primero. En términos generales, la regla lex posterior presupone fuentes del mismo valor jerárquico e identidad de destinatarios de las normas, es decir que no puede producir sus efectos más que con relación a las partes que sean las mismas en los dos tratados. En este caso, se presume que el tratado posterior manifiesta la nueva intención legislativa de las partes contratantes y, por lo tanto, tendrá prioridad. De esta forma, la posterioridad de un tratado otorga a este un título de aplicación preferente basado en una presunción de mayor eficacia.

36 En este sentido, la redacción del artículo 30 difiere de la de muchas otras disposiciones de la Convención de Viena que aparecen redactadas según la famosa fórmula de filiación anglosajona, "salvo que el tratado disponga otra cosa", que no deja lugar a dudas acerca de su carácter supletorio — se aplican en ausencia de régimen convencional expreso—. 
Por otro lado, no hay ninguna dificultad, a nuestro entender, en admitir que el párrafo 3 deja abierta la posibilidad a que formen parte del segundo tratado Estados que no lo fueron del primero, aunque, en cambio, creemos que sería más dudoso convenir que en este contexto de sucesión llegasen a ser parte del tratado anterior nuevos Estados. De este modo, estos terceros Estados en relación con el primero no quedarían pues afectados por la aplicación de la regla lex posterior que rige solo entre aquellos que lleguen a ser parte en ambos tratados.

Sin duda alguna, el punto más controvertido de la solución propuesta en el párrafo tercero radica en la identificación de la 'compatibilidad/ incompatibilidad' entre el tratado posterior y el tratado anterior, pues el artículo 30 prevé la aplicación del tratado anterior en la medida en que sus disposiciones no sean incompatibles con las del posterior, lo cual supone efectuar una comparación entre ambos tratados.

En esta línea, al celebrarse la $91^{\text {a }}$ sesión de la CDI, el presidente del Comité de Redacción, M. Yasseen, esclareció en su informe las posibles interpretaciones con relación a los conceptos de compatibilidad e incompatibilidad expresados en el párrafo 3 del entonces artículo 26. Lo hizo afirmando que la mera circunstancia de que lo dispuesto en el tratado posterior pudiera divergir de lo establecido en el anterior no implica, necesariamente, incompatibilidad. Así mismo, y a raíz de un requerimiento soviético ante este Comité, en el sentido de que el artículo no debía impedir la subsistencia de un tratado anterior si su régimen era más liberal para las partes que el tratado posterior, el presidente del Comité expresó:

En realidad el mantenimiento de la vigencia de las disposiciones de un tratado anterior pudiera justificarse en razón de las circunstancias o de la intención de las partes. Así acontecería, por ejemplo, en el siguiente caso: si un pequeño grupo de Estados celebrara una convención en materia consular, en la que se otorgaran amplios privilegios e inmunidades, y si posteriormente esos mismos Estados celebraran con otros Estados una convención en materia consular, en la que el número de partes fuera mucho mayor, pero en la cual se estableciera un régimen más restringido, la convención anterior continuaría rigiendo las relaciones entre los Estados partes en la misma, si las circunstancias o la intención de las partes justificaran el que se la mantuviera en vigor. ${ }^{37}$

37 A/CONF.39/C.1/15. 
Lo anteriormente expuesto nos lleva a concluir que la identificación de la compatibilidad entre las disposiciones convencionales no es sino un problema de interpretación de estas, habida cuenta de que la función que va a desarrollar esta es la de determinación y precisión del sentido y alcance de las disposiciones convencionales, a los fines de establecer el consenso de los Estados expresado en el tratado. Para ello, deberá atenderse a las reglas de interpretación de los tratados recogidas en los artículos 31 a 33 de la Convención de Viena de 1969.

Pero en este contexto concreto debemos además tener igualmente presente que, como muy bien advierte el profesor Remiro Brotóns, "siempre es preciso determinar el sentido, fijar el alcance, esclarecer los puntos oscuros y las ambigüedades — tal vez deliberadamente deslizadas por los negociadores - de una disposición, antes de ordenar sus consecuencias en el caso concreto". ${ }^{38}$ Esta tarea se hace imperiosamente necesaria en el marco de la aplicación de tratados sucesivos concernientes a la misma materia que plantean hipotéticos problemas de compatibilidad entre sus disposiciones. Circunstancia que viene a corroborar la tesis de que la interpretación de las normas internacionales suele plantearse de forma generalizada — aunque no exclusiva — con ocasión de su aplicación al caso concreto, pues es cuando se aplica la norma a un supuesto determinado que pueden surgir las controversias entre las partes afectadas.

Una última puntualización. Antes de aplicar la solución en favor del tratado posterior que propone el párrafo tercero, habrá lugar de verificar si por la celebración de un nuevo tratado sobre la misma materia entre las mismas partes estas han decidido suprimir definitivamente o suspender de forma temporal la aplicación del tratado anterior. Pues, en este caso, no se tratará de fijar el orden de prioridades entre disposiciones convencionales según el artículo 30.3, sino de determinar si hay terminación o suspensión tácita de la aplicación del tratado anterior según lo dispuesto por el artículo 59 de la Convención de 1969.

No hay duda de que existe una estrecha relación entre los artículos 30.3 y 59; en ambos casos estamos situados ante tratados sucesivos concernientes a la misma materia, y las dos disposiciones regulan el supuesto de identidad de partes en ambos tratados sucesivos. En todo caso, la coexistencia actual de los artículos 30.3 y 59 en la Convención de Viena

38 Remiro Brotóns, A., Derecho internacional público. 2. Derecho de los tratados, Tecnos, Madrid, 1987, p. 307. 
conduce finalmente a la siguiente situación: en caso de imposibilidad de aplicación de dos tratados al mismo tiempo, se deberá verificar, en primer lugar, si las partes tienen intención de revocar o suspender la aplicación del primer tratado; si la respuesta es afirmativa, es el elemento subjetivo el que vence; en caso negativo, una presunción objetiva de divisibilidad entra en juego. En los dos casos se favorece al tratado posterior. Pero, por lo que se refiere a las relaciones entre el artículo 30.3 y el artículo 59.1.b), la diferencia radica en el alcance de la incompatibilidad existente entre los dos tratados: si la incompatibilidad es total, se produce la abrogación también total del tratado anterior (artículo 59.1.b); pero tratándose de una incompatibilidad parcial o relativa, se permite la subsistencia igualmente parcial o relativa del tratado anterior, que continuará aplicándose "en la medida de su compatibilidad con el tratado posterior" (artículo 30.3). Esta situación avoca, pues, a una imperativa comparación previa entre ambos tratados que determine el grado de 'incompatibilidad'.

Realmente, la línea que separa a uno de otro viene marcada, nuevamente, por una simple cuestión de interpretación.

\subsection{No identidad de partes en los tratados}

El párrafo 4 del artículo 30 se ocupa de algunos problemas aún más complejos que surgen en el caso en que ciertas, pero no todas las partes de un tratado anterior, son partes en el posterior tratado sobre la misma materia, esto es, cuando no se produce identidad de partes en ambos tratados. Se trata, sin duda, del aspecto más difícil de todas las cuestiones relativas a la aplicación de tratados sucesivos concernientes a la misma materia, concretamente, las relaciones de los Estados parte en los dos tratados respecto de Estados que son terceros con relación a uno de los tratados en juego, pues, junto al principio de autonomía de la voluntad, se debe introducir otro principio que va a combinarse con él, el de la relatividad de los tratados, que se traduce en la ausencia de efecto de los tratados con relación a terceros. Tales conflictos pueden surgir tanto en la esfera bilateral (A está obligado a observar cierta conducta por un tratado con B, y concluye otro tratado con $\mathrm{C}$ por el que se vincula respecto de un comportamiento incompatible con el fijado por el tratado entre A y B) como en la multilateral (ABCDE y ABWYZ).

El párrafo 4 tiende a crear dos regímenes convencionales diferentes interpartes. 
a) en las relaciones entre los Estados parte en ambos tratados se aplicará la norma enunciada en el párrafo 3;

b) en las relaciones entre un Estado que sea parte en ambos tratados y un Estado que solo lo sea en uno de ellos, los derechos y obligaciones recíprocos se regirán por el tratado en el que los dos Estados sean partes.

Esta regla de solución propuesta impide que las partes en el tratado posterior priven a las demás partes en el tratado anterior — sin su consentimiento- de los derechos que les correspondían conforme a ese tratado. Se trata de mantener un absoluto respeto a la regla pacta tertiis nec nocent nec prosunt, pues las partes del tratado anterior que no lo son en el posterior son terceros respecto de este, en la misma medida en que lo son las partes del posterior que no ostentan tal carácter respecto del anterior. De esta forma, esta disposición fragmenta las relaciones jurídicas en dos esferas diferentes:

1. Para el supuesto de las relaciones entre Estados que son partes en los dos tratados, el párrafo 4 a) reenvía a la solución prevista en el párrafo 3, esto es, la aplicación del criterio de la lex posterior, bajo reserva evidentemente del artículo 59. Es decir, la norma enunciada en este párrafo a) no constituye más que una aplicación del principio general del que ha de presumirse que la expresión posterior de la intención de las partes prevalecerá sobre la anterior.

2. Pero las relaciones entre un Estado parte en uno solo de los tratados sucesivos - ya sea del tratado anterior, ya sea del posterior- y un Estado parte en los dos tratados concurrentes deberán estar regidas por aquel tratado en el que participen ambos Estados, que puede ser tanto el anterior como el posterior. Nos enfrentamos, pues, a una aplicación particular de la norma enunciada en el artículo 30 que es el resultado de la conjugación de los principios pacta sunt servanda, relatividad de los tratados y pacta tertiis nec nocent nec prosunt; no se puede aplicar un tratado a Estados que son terceros respecto de este.

Es importante hacer notar que el texto evita el uso de los términos 'prevalece' o 'se aplicará', que, en cambio, sí aparecen en los párrafos 
precedentes. ${ }^{39}$ A nuestro entender, la razón que justifica esta - por otro lado lógica- ausencia es que dichas expresiones presuponen un juicio de valor que podría conducir a hacer debilitar, o incluso invalidar, a aquel tratado en el que no participen todas las partes.

Una situación como la prevista en el párrafo 4 es la que se produce entre la CNUDM de 1982 y el ya citado Acuerdo de Nueva York de 1995. Pues sucede que, tras la aparente bondad de su propio título y los buenos propósitos de la cláusula relacional de compatibilidad del artículo $4^{\circ}$ de este último — que hemos visto—, resulta que el Acuerdo de 1995 contiene varias incompatibilidades con relación a la CNUDM; incluso, podríamos llegar a afirmar que el Acuerdo constituye pura y llanamente una alteración de ciertas normas consolidadas de la Convención concernientes a la pesca en alta mar. ${ }^{40}$ Siendo esto así, y dado que no hay identidad de partes entre ambos tratados sucesivos, por lo que a esta cuestión material se refiere deberá aplicarse la regla prevista por el artículo 30.4. Todo ello sin perjuicio de las posibles consecuencias jurídicas que pudiere conllevar tanto la aplicación como la celebración de este acuerdo incompatible con la CNUDM —en la medida en que puede entrar en contradicción con lo dispuesto en el artículo 311.4 de esta-, según lo dispuesto en el artículo 30.5 de la Convención de 1969.

Por otra parte, creemos necesario señalar que, si bien el criterio apuntado en el párrafo 4 supone una solución coherente con los más básicos principios del derecho de los tratados, debemos tener en cuenta que esta disposición está fundada sobre la hipótesis de que se pueden distinguir y tratar separadamente dos órdenes de relaciones jurídicas: aquella entre Estados parte en los dos tratados, y las de aquellas entre un Estado parte

39 El término 'prevalece' había sido propuesto por el relator especial en su proyecto de 1964. Sin embargo, tanto Verdross como Yasseen sugirieron reemplazarlo por el más genérico de 'se aplicará', que fue el recogido en el proyecto definitivo de 1966 (Anuario de la CDI, 1966-I, 742 a sesión, párrs. 16-17, y 743ª sesión, párr. 61). Sin embargo, la formulación final 'se regirán' fue introducida por el Comité de Redacción.

40 A este respecto, las contradicciones y modificaciones más reseñables que comporta el Acuerdo de 1995 respecto de la Convención de 1982 son las contenidas en los artículos 3.1, 3.2, 8.4, 17.2, 18.1, 18.2, 21.3, 21.8, 22 —especialmente el 22.1.f), que puede incluso colisionar con la norma imperativa relativa a la prohibición del uso de la fuerza-. Un análisis en detalle de los conflictos convencionales en el derecho del mar, incluido el que reseñamos, puede consultarse en nuestro trabajo López Martín, A. G., "Conflicto entre tratados: tempestad o calma en el derecho del mar", Foro, Nueva Época, 2006, 3, pp. 241-278. 
en los dos tratados y un Estado parte en uno de los tratados solamente. Si esta distinción se considera admitida, las relaciones del primer grupo deben estar determinadas como se ha indicado más arriba, reduciéndose el problema a una simple cuestión de interpretación de la voluntad de las partes; mientras las relaciones del segundo grupo se rigen por aquel tratado en el que los dos Estados son partes.

Pero la cuestión esencial es la de saber en qué condiciones es factible una tal descomposición en dos órdenes de relaciones jurídicas. La redacción del texto muestra que es necesario que los dos tratados sean compatibles con esta descomposición, y, si esto no es posible, que los tratados multilaterales en causa —al menos en uno de los dos que es multilateral - se dejen descomponer en una serie de acuerdos bilaterales independientes sin perder su razón de ser. Tal parece ser el caso de una convención consular multilateral o de una convención que tenga por objeto unificar reglas nacionales de derecho privado (al menos en la mayor parte de los casos). Pero no se puede afirmar que sea siempre así, pues, pasando el marco de la simple reciprocidad, ciertos tratados multilaterales se resisten a la descomposición en un haz de acuerdos bilaterales; y, si esta condición no se cumple, el segundo tratado aparecerá como violación del primero, o, si se prefiere, la ejecución del tratado posterior conducirá a impedir la ejecución del tratado anterior, es entonces cuando entra en juego la solución final apuntada por el párrafo 5 del artículo 30 que abordaremos a continuación.

Apuntar, finalmente, que este mismo párrafo 5 formula una reserva respecto de las disposiciones del artículo 41 de la Convención de Viena, relativas a la modificación inter se de los tratados multilaterales, pues nada de lo dispuesto en el párrafo 4 habrá de entenderse de manera que excluya dichas estipulaciones.

\section{Consecuencias jurídicas de la existencia de tratados incompatibles}

Tal y como se acaba de advertir, la aplicación de las reglas del artículo 30.4 puede no solucionar sin más el conflicto, de tal forma que un Estado, al celebrar o aplicar un tratado incompatible con otro, incurra en violación respecto de otro. Lo que conlleva que todas las consecuencias normales de la violación de los tratados se producirán respecto a dicho Estado. Así lo establece el artículo 30.5: "El párrafo 4 [...] no prejuzgará ninguna cuestión de terminación o suspensión de la aplicación de un tratado conforme al 
artículo 60 ni ninguna cuestión de responsabilidad en que pueda incurrir un Estado por la celebración o aplicación de un tratado cuyas disposiciones sean incompatibles con las obligaciones contraídas con respecto a otro Estado en virtud de otro tratado".

La conclusión que cabe extraer de la lectura de esta disposición es que, una vez materializado un conflicto de tratados irresoluble por los criterios propuestos por el artículo 30, carecemos de una regla de conflicto al efecto, pues el párrafo 5 no ofrece realmente ninguna solución específica para esta situación. Se limita a realizar una doble remisión a la normativa internacional pertinente que es de aplicación para el supuesto en el que un Estado viole alguna de las obligaciones convencionales contenidas en alguno de los tratados sucesivos de los que es parte, ante la incompatibilidad manifiesta existente entre ellas. Por un lado, al artículo 60 de la Convención que se refiere a la posibilidad de dar por terminado o suspendido el tratado por violación grave de este. Por otro, que no es excluyente de lo anterior, a las normas sobre responsabilidad internacional.

Pero ¿a qué llamamos conflicto irresoluble? Enfrentados a dos tratados sucesivos concernientes a la misma materia, uno debe mirar si existen cláusulas de relación que resuelvan el conflicto; en ausencia de cláusulas, habrá de proceder a la interpretación de los tratados en cuestión, sobre la base de los artículos 31 y 32 de la Convención de 1969, en busca de la voluntad de las partes acerca de la prioridad de los tratados; cuando una tal interpretación resulta inconclusa, se debe recurrir a la lex posterior, que no plantea problema tratándose de una hipótesis de identidad de partes. En estos supuestos, el conflicto se resuelve y, por lo tanto, la responsabilidad no entra en juego.

Pero, cuando no es el caso, esto es, cuando se produce la celebración de un tratado posterior sobre la misma materia expresamente prohibido por el tratado anterior - ya sea de modificación, de enmienda, de suspensión o simplemente de manifestación de una nueva intención legislativa entre algunas partes, bien solas, bien con terceros Estados_-; o la aplicación de sus disposiciones viola los derechos de otras partes del tratado anterior; o cuando las disposiciones del tratado posterior infringen seriamente las del primero, que son indispensables para la efectiva conclusión del objeto y fin de dicho tratado; o bien no es posible proceder a la descomposición de relaciones convencionales en los términos propuestos por el artículo 
30.4 de la Convención, entonces existe conflicto 'irresoluble'.$^{41}$ En estos casos, se puede decir que dos principios fundamentales han colisionado: pacta sunt servanda y pacta tertiis nec nocent nec prosunt.

El Estado que se halla inmerso en tal conflicto de obligaciones convencionales debe decidirse por aplicar uno de los tratados, con lo que inevitablemente viola el otro. Si tal sucede, la parte perjudicada podrá invocar su derecho a poner término al tratado o a suspender su aplicación, cuando se trate de una violación grave conforme al artículo 60 de la Convención de 1969. Y podrá también alegar la responsabilidad internacional de la parte que haya violado sus derechos, lo que deberá traducirse en la cesación del comportamiento ilícito y en la reparación del daño. Para ello podrá hacer uso de las contramedidas en los términos y condicionantes del proyecto de la CDI sobre responsabilidad internacional de los Estados por hechos ilícitos de 2001, pudiendo el Estado perjudicado optar tanto por la aplicación de la exceptio non adimpleti contractus en sentido estricto, como por no cumplir obligaciones previamente contraídas para con el Estado infractor.

Sin duda, prevalece en este ámbito el principio de voluntad política; el Estado es quien decide qué tratado aplicará y, en consecuencia, cuál va a ser violado. Tal es así, porque, frente a teorías clásicas que sostenían la invalidez o la nulidad del tratado posterior que entraba en conflicto con el tratado anterior, ${ }^{42}$ la posición imperante hoy día es la del mantenimiento de los dos tratados perfectamente válidos. Este es el espíritu que se desprende del artículo 30 de la Convención.

\section{Una valoración final}

Advertíamos al inicio de este trabajo que los conflictos entre tratados que regulan la misma materia constituyen uno de los aspectos más complejos y

41 Como hemos indicado, pudiera ser el caso del Acuerdo de Nueva York de 1995 con relación a la CNUDM de 1982, tanto por su celebración (CNUDM, art-311.4) como por su aplicación.

42 Tal es el caso de Gentilis, Grocio, Pufendorf o Vattel. Si bien la doctrina clásica ya fue cuestionada desde un primer momento por el Proyecto de Convención sobre Derecho de los Tratados de la Harvard Law School de 1935 (AJIL, 1935, supl., pp. 653 y ss.). Aunque hemos de advertir que el primer relator de la CDI que abordó el problema del conflicto de tratados, Lauterpacht, retomó en sus dos informes la teoría clásica que, finalmente, fue rechazada (Yearbook of ILC, 1953-II, pp. 90 y ss., y pp. 156-159). 
oscuros del derecho de los tratados. Siendo los conflictos convencionales más abundantes en la realidad de la práctica de lo que una concepción cartesiana del derecho internacional podría considerar comprensible.

El análisis pormenorizado de la disposición que establece las reglas para solucionarlos no hace sino corroborar dicha valoración.

Tal y como se ha podido advertir, bajo su aparente simplicidad, el artículo 30 esconde una casuística compleja, plagada de remisiones y reenvíos. De igual forma, presenta unas innegables deficiencias técnicas congénitas, por no hablar de la existencia de lagunas importantes en dicha regulación, como es la ausencia de disposición alguna que recoja una mínima referencia al criterio de la lex specialis; circunstancia que resulta ciertamente paradójica si tenemos presente el papel tan significativo que opera dicha regla en el derecho de los tratados.

Todo ello obstaculiza enormemente la adecuada interpretación de sus reglas de aplicación, al tiempo que no aporta más que elementos parciales a la solución de tan intrincada cuestión, lo cual se hace aún más evidente en aquellos supuestos diabólicos caracterizados por la inexistencia de cláusula relacional, bien porque los Estados no han sabido, bien porque no han querido incluirla; situación que no es ni mucho menos excepcional. Pues es entonces cuando se muestra con mayor crudeza la inherente dificultad de la solución de los conflictos convencionales.

Siendo además que, situados sobre el plano práctico, las reglas del artículo 30 no resuelven todos los supuestos de aplicación de tratados sucesivos concernientes a la misma materia. Pues, en ocasiones, los conflictos convencionales ocultan silenciosamente otros conflictos intranormativos implícitos y buscados expresamente. Lo que, sin duda, dificulta enormemente la labor del jurista.

\section{Referencias}

Annuaire de l'IDI, Sesión de Roma, 1973.

Anuario de la CDI, 1964-I, $2^{\mathrm{a}}$ parte.

Anuario de la CDI, 1966-I.

Anuario de la CDI, 1966-II.

Aust, A., Modern treaty law and practice, Cambridge University Press, 2007.

Beckett, W. E., The North Atlantic Treaty, the Brussels Treaty and the Charter of the United Nations, London, 1950.

Bernhardt, R., "Article 103", en Simma, Bruno (ed.), The Charter of the United Nations: a commentary, Oxford University Press, 1994, pp. 2120 y ss. 
Conferencia de las Naciones Unidas sobre el Derecho de los Tratados, Nueva York, 1969.

Conforti, B., "Organizzazione delle Nazioni Unite”, en Enciclopedia del diritto, vol. XXXI, Torino, 1981.

Corten, O. \& Klein, P. (Eds.), The Vienna Conventions on the Law of Treaties. A commentary, vol. I, Oxford University Press, 2011.

Czaplinski, W. \& Danilenko, G., "Conflicts of norms in international law", NYIL, 1990, pp. 5 y ss.

Dahl, K. N., "The application of successive treaties dealing with the same subject matter", Indian Yearbook of International Affairs, 1974, pp. 280 y ss.

Giardina, A., “The Egytptian-Israeli Peace Agreement”, Italian Yearbook of International Law, 1979, pp. 21 y ss.

Jenks, L. W., “The conflict of law-making treaties”, BYBIL, 1974-75, pp. 440 y ss.

Kolb, R., "L'article 103 de la Charte des Nations Unies", Recueil des Cours, 2013, 367, pp. 1 y ss.

López Martín, A. G., "Conflicto entre tratados: tempestad o calma en el derecho del mar”, Foro, Nueva Época, 2006, 3, pp. 241-278.

Meron, T., "On a Hierarchy of international human rights", AJIL, 1986, pp. 1 y ss.

Paolillo, F., "Article 30", en Corte, Olivier \& Klein, Pierre (dirs.), Les Conventions de Vienne sur le droit des traités. Commentaire article par article, Bruylant, Bruxelles, 2006, pp. 1259 y ss.

Paulus, L., "Article 103", en Simma, Bruno (ed.), The Charter of the United Nations. A commentary, vol. II, $3^{\text {a }}$ ed., Oxford University Press, 2012, pp. 2115 y ss.

Picone, P., L’applicazione in via provisoria degli accordi internazionali, Nápoles, 1973.

Remiro Brotóns, A., Derecho internacional público. 2. Derecho de los tratados, Tecnos, Madrid, 1987.

Reuter, P., Introduction au droit des traités, París, 1985.

Roucounas, E., "Engagements paralléles et contradictoires", Recueil des Cours, 1987-VI, 206, pp. 4 y ss.

Sciso, E., Gli Accordi Internazionali Confliggenti, Bari, 1986.

Sinclair, I., The Vienna Convention on the Law of Treaties, Manchester, 1984.

Vierdag, E. W., “The time of the 'conclusion' of a multilateral treaty: article 30 of the Vienna Convention on the Law of Treaties and related provisions", BYBIL, 1988, pp. 90 y ss. 
Yearbook of ILC, 2004-II.

Yearbook of ILC, 1953-II. 